\title{
Numerical Study of One Dimensional Fishers KPP Equation with Finite Difference Schemes
}

\author{
Shahid Hasnain, Muhammad Saqib, Daoud Suleiman Mashat \\ Department of Mathematics, Numerical Analysis, King Abdulaziz University, Jeddah, KSA \\ Email:shahidqa32@gmail.com,dmashat@kau.edu.sa
}

How to cite this paper: Hasnain, S., Saqib, N. and Mashat, S. (2017) Numerical Study of One Dimensional Fishers KPP Equation with Finite Difference Schemes. American Journal of Computational Mathematics, 7, 70-83.

https://doi.org/10.4236/ajcm.2017.71006

Received: December 30, 2016

Accepted: March 28, 2017

Published: March 31, 2017

Copyright $\odot 2017$ by authors and Scientific Research Publishing Inc. This work is licensed under the Creative Commons Attribution International License (CC BY 4.0).

http://creativecommons.org/licenses/by/4.0/ (c) (i) Open Access

\begin{abstract}
In this paper, we originate results with finite difference schemes to approximate the solution of the classical Fisher Kolmogorov Petrovsky Piscounov (KPP) equation from population dynamics. Fisher's equation describes a balance between linear diffusion and nonlinear reaction. Numerical example illustrates the efficiency of the proposed schemes, also the Neumann stability analysis reveals that our schemes are indeed stable under certain choices of the model and numerical parameters. Numerical comparisons with analytical solution are also discussed. Numerical results show that Crank Nicolson and Richardson extrapolation are very efficient and reliably numerical schemes for solving one dimension fisher's KPP equation.
\end{abstract}

\section{Keywords}

Forward in Time and Centre in Space (FTCS), Lax Wendroff, Taylor's Series, Crank Nicolson and Richardson Extrapolation

\section{Introduction}

Fisher gives introduction to nonlinear evolution equation to inquisitive, the proliferation of an beneficial gene in a population dynamics [1]. Fisher's equation also specify the logistic diffusion process [1]. It has the form

$$
u_{t}=\beta u_{x x}+\alpha u(1-u)
$$

where $\beta>0$ is a diffusion constant with $\alpha>0$ is the linear growth rate [1]. The reaction diffusion Equation (1) also express a model equation for the evolution of a neutron population in a nuclear reactor [2] and also appears in chemical engineering applications [2]. This equation accommodates the effects of linear diffusion along $u_{x x}$ and nonlinear local multiplication or reaction along $u(1-u)$ [3] [4]. It has become one of the most important nonlinear equations and occurs in many biological and chemical processes [4] [5]. Recently, many researcher are working on this type of model to understand growth rate 
and diffusion aspect, for example, Abdullaev has studied the stability of symmetric travelling waves in the Cauchy problem for a more general case than Equation (1) [6]. Also Logan has studied this problem using a perturbation method and settled up with an approximate solution by expanding the solution in terms of a power series and in terms of some small parameter [7]. The finite difference schemes and auxiliary conditions of the numerical model must be consistent with the partial differential equations and initial and boundary conditions of the mathematical model [8] [9]. The numerical model is consistent if the truncation error, that is the discrepancy between the finite difference approximation and the continuous derivatives, tends to zero as the grid spacing get smaller and smaller. Secondly the solution to finite difference schemes must converge to the solution of the partial differential equations as the grid spacing gets smaller and smaller [9]. Thus we can say that, difference between the exact solution and approximated solution must vanish as the grid spacing tends to zero.

In this paper, we started the solution of Fishers equation with various finite difference schemes. We discussed Forward in time and Central in space (FTCS) and Lax-Wendroff schemes, which are explicit and conditionally stable. Also FTCS is first order accurate in time and second order accurate in space and Lax-Wendroff is second order accurate in both space and time, which shows improvement in accuracy in later method. Crank-Nicolson scheme is unconditionally stable and implicit with second order accuracy in both space and time [9]. This applies computational stability for any size of the time increment [9]. However, the size of $\Delta t$ is still limited by accuracy required in the solution. Usage of very large values of time step results in poor approximation to solution because of unacceptably large truncation errors produced [10]. We applied Richardson extrapolation method to improve accuracy with no issue of stability. We came to know this method is highly accurate and easy to implement with no mess of computations [10] [11] and we can see from our results that this method is excellent agreement to the exact solution.

\section{Exact Solution}

Let us consider Equation (1), within domain $x \in(-\infty, \infty)=\Omega$ with $t \geq 0$. To derive the exact solution of the given Equation (1), we have the following exact solution [10] [11] to above equation,

$$
\begin{aligned}
& \left.\begin{array}{c}
\left.\begin{array}{c}
D=\sqrt{\alpha / 6} x-(5 / 6) \alpha t \\
u(x, t)=1 /\left(1+e^{D}\right)^{2}
\end{array}\right\}
\end{array}\right\} \\
& \text { subject to initial condition, } \\
& \left.\begin{array}{r}
D_{1}=\sqrt{\alpha / 6} x \\
u(x, 0)=1 /\left(1+e^{D_{1}}\right)^{2}
\end{array}\right\} \\
& \text { Boundary Conditions, } t>0, x \in \Omega \\
& \left.u(0, x)=u_{0}(x)\right\} \\
& \text { initial Conditions, } x \in \Omega \text {. }
\end{aligned}
$$




\section{Numerical Methods}

We consider the numerical solution of the non-linear Equation (2) in a finite domain $\Omega$. The first step is to choose integers $n$ to define step sizes $h=(b-a) / n$. Partition the interval $[a, b]$ into $n$ equal parts of width $h$. Place a grid on the rectangle $\mathrm{R}$ by drawing vertical and horizontal lines through the points with coordinates $\left(x_{i}\right)$, where $x_{i}=a+i$ for each $i=0,1,2, \cdots n$ also the lines $x=x_{i}$ represent grid lines, also we assume $t_{n}=n t, n=0,1, \cdots$ where $t$ is the time grid step size. We denote the exact and numerical solutions at the grid point $\left(x_{m}, t_{n}\right)$ by $u_{m}^{n}$ and $U_{m}^{n}$ respectively. We consider four finite different schemes as we mentioned in keywords in abstract.

\subsection{FTCS Scheme}

We consider forward in time and center in space (FTCS) explicit scheme by substituting the forward difference approximation for the time derivative and the central difference approximation for the space derivative in Equation (1),

$$
\begin{gathered}
u=u_{i}^{n} \\
u_{t}=\frac{u_{i}^{n+1}-u_{i}^{n}}{k} \\
u_{x x}=\frac{u_{i+1}^{n}-2 u_{i}^{n}+u_{i-1}^{n}}{h^{2}}
\end{gathered}
$$

which leads to the following,

$$
u_{i}^{n+1}=u_{i}^{n}+\boldsymbol{R}_{1}\left(u_{i+1}^{n}-2 u_{i}^{n}+u_{i-1}^{n}\right)+k u_{i}^{n}\left(1-u_{i}^{n}\right)
$$

where $\boldsymbol{R}_{1}=\frac{k}{h^{2}}$, final form of above equation is,

$$
u_{i}^{n+1}=u_{i}^{n}\left(1+k-2 \boldsymbol{R}_{1}-k u_{i}^{n}\right)+\boldsymbol{R}_{1}\left(u_{i+1}^{n}+u_{i-1}^{n}\right) .
$$

Since the one dimensional diffusion reaction equation is nonlinear and wellposed [10] [11], Lax's equivalence theorem indicates that consistency and stability of the FTCS finite difference approximation is necessary and sufficient for FD solution to converge to diffusion reaction equation [12]. Once convergence has been proved, the solution to the given partial differential equation can be obtained to any desired degree of accuracy [12]. Make sure the spacing $h$ for spatial and $k$ for time of the finite difference grid are made sufficiently small. The FTCS scheme, from Equation (3), is classified as explicit because the value of $u_{i}^{n+1}$ at the $(n+1)$ th time level may be calculated directly from known value of $u_{i}^{n}$ at previous time levels. It is a two level method because values of $u$ at only two levels of time are involved in the approximating finite difference equation [12].

\section{Accuracy and Stability to FTCS Scheme:}

Accuracy:

To find accuracy of the FTCS scheme for Fisher-KPP equation, we apply Taylor's series on each term of the equation. Let us consider the Taylor's series in the following way, 


$$
\begin{gathered}
u_{t}=u_{i}^{n+1}-u_{i}^{n} \\
u_{t}=u(x, t+k)-u(x, t) \\
\text { Apply Taylor's series } \\
u_{t}=k u_{t}+\frac{k^{2}}{2} u_{t t}+\frac{k^{3}}{6} u_{t t t}+\cdots \\
u_{x x}=u_{i+1}^{n}-2 u_{i}^{n}+u_{i-1}^{n} \\
u_{x x}=u(x+h, t)-2 u(x, t)+u(x-h, t) \\
\text { Apply Taylor's series } \\
u_{x}=h^{2} u_{x x} \frac{h^{4}}{12} u_{x x x}+\cdots
\end{gathered}
$$

Take Equation (1) into account, and apply this scheme with Taylor's series on each term, updated equation is as follows.

$$
\begin{aligned}
E q .= & u(x, t+k)-u(x, t)-\boldsymbol{R}_{1}(u(x+h, t)-2 u(x, t) \\
& +u(x-h, t))-k u(x, t)(1-u(x, t)) \\
\text { Eq. }= & \left(u_{t}-u_{x x}-u(1-u)\right) k+\frac{1}{2} u_{t t} k^{2}-\boldsymbol{R}_{1} u_{x x} h^{2}+\frac{1}{6} u_{t t t} k^{3} \\
& -\frac{1}{12} \boldsymbol{R}_{1} u_{x x x x} h^{4}+\cdots
\end{aligned}
$$

Now principle part of the truncation error is along with Equation (1). So first part of the above equation goes to zero if we consider Equation (1).

$$
\text { PPTE of } u=\frac{1}{2} u_{t t}-\boldsymbol{R}_{1} u_{x x} h^{2}+\frac{1}{6} u_{t t t} k^{2}-\frac{1}{12} \boldsymbol{R}_{1} u_{x x x x} h^{4}+\cdots \text {. }
$$

Which shows that this scheme is first order accurate in time and 2nd order accurate in space, such as $O\left(k, h^{2}\right)$.

Stability:

We want to study under what condition the error can be magnified. Many methods can be used to study this issue. We consider only Von-Neumann stability analysis to explain this method on FTCS scheme. Consider the scheme in the following way,

$$
u_{m}^{n+1}=u_{m}^{n}+\boldsymbol{R}_{1} \delta_{x}^{2} u_{m}^{n}+k u_{m}^{n}\left(1-u_{m}^{n}\right) .
$$

Linear form of the above equation is,

$$
u_{m}^{n+1}=u_{m}^{n}+\boldsymbol{R}_{1} \delta_{x}^{2} u_{m}^{n}+k u_{m}^{n}(1-\text { Constant }) .
$$

According to Von-Neumann stability analysis, let us consider the solution as:

$$
U_{m}^{n}=e^{\alpha n k} e^{i \beta m h} .
$$

The Von-neumann stability condition is

$$
\left|e^{\alpha k}\right| \leq 1
$$

Note that,

$$
\begin{aligned}
U_{m}^{n+1} & =e^{\alpha(n+1) k} e^{i \beta m h} \\
U_{m}^{n-1} & =e^{\alpha(n-1) k} e^{i \beta m h} \\
U_{m+1}^{n} & =e^{\alpha(n) k} e^{i \beta(m+1) h} \\
U_{m-1}^{n} & =e^{\alpha(n) k} e^{i \beta(m-1) h} .
\end{aligned}
$$


Also

$$
\begin{aligned}
& \delta_{x}^{2} U_{m}^{n}=-4 \sin ^{2}\left(\frac{\beta h}{2}\right)\left[e^{\alpha n k} e^{i \beta m h}\right] \\
& \delta_{x}^{2} U_{m}^{n+1}=-4 \sin ^{2}\left(\frac{\beta h}{2}\right)\left[e^{\alpha(n+1) k} e^{i \beta m h}\right] .
\end{aligned}
$$

Apply above terms to Equation (1), we get the following

$$
e^{\alpha k}=1-4 \boldsymbol{R}_{1} \sin ^{2}\left(\frac{\beta h}{2}\right)+k(1-\text { Constant }) .
$$

According to Equation (6),

$$
-1 \leq e^{\alpha k} \leq 1 .
$$

Take left hand side of above equation along Equation (7),

$$
\begin{aligned}
& 1-4 \boldsymbol{R}_{1} \sin ^{2}\left(\frac{\beta h}{2}\right)+k(1-\text { Constant }) \leq 1 \\
& \Rightarrow-4 \boldsymbol{R}_{1} \sin ^{2}\left(\frac{\beta h}{2}\right) \geq k(1-\text { Constant }) \\
& \max \left(\sin ^{2}\left(\frac{\beta h}{2}\right)\right)=1 \\
& \boldsymbol{R}_{1} \geq \frac{k(1-\text { Constant })}{4} \\
& \text { If we choose Constant }=0 \\
& \boldsymbol{R}_{1} \geq \frac{k}{4} .
\end{aligned}
$$

Take right hand side of above equation along Equation (7),

$$
\begin{aligned}
& -1 \leq 1-4 \boldsymbol{R}_{1} \sin ^{2}\left(\frac{\beta h}{2}\right)+k(1-\text { Constant }) \\
& \Rightarrow \boldsymbol{R}_{1} \leq \frac{2+k(1-\text { Constant })}{4} \\
& \text { If we choose Constant }=0 \\
& \boldsymbol{R}_{1} \leq \frac{2+k}{4} .
\end{aligned}
$$

Since $\boldsymbol{R}_{1}=k / h^{2}$, according to Von-Neumann stability analysis on both sides as left and right, which shows that FTCS scheme is conditionally stable for Fisher-KPP equation.

\subsection{Lax Wendroff Scheme}

A numerical technique proposed in 1960 by P.D. Lax and B. Wendroff [13] [14] [15] for solving partial differential equations and system numerically. In spite of the impressive developments on numerical methods for partial differential equations from 1970s onwards, in which the Lax Wendroff method has played a historic role, there are presently (1998) substantial research activities aimed at further improvements of methods [15]. Lax Wendroff's method is also explicit method but needs improvement in accuracy in time. This method is an example of explicit time integration where the function that defines governing equation is 
evaluated at the current time [15]. Purpose of this method to achieve good enough accuracy in time. This method can be explained in two steps which is as follows,

$$
u_{i}^{\star}=0.5\left(u_{i+1}^{n}+u_{i-1}^{n}\right)-0.5 \boldsymbol{R}_{1}\left(u_{i+1}^{n}-2 u_{i}^{n}+u_{i-1}^{n}\right)+0.5 \alpha k u_{i}^{n}\left(1-u_{i}^{n}\right)
$$

2nd step is

$$
u_{i}^{n+1}=u_{i}^{\star}+\boldsymbol{R}_{1}\left(u_{i+1}^{\star}-2 u_{i}^{\star}+u_{i-1}^{\star}\right)+0.5 \alpha k u_{i}^{\star}\left(1-u_{i}^{\star}\right)
$$

\section{Accuracy and Stability to Lax-Wendroff Scheme:}

Accuracy:

To find accuracy of the Lax-Wendroff scheme for Fisher-KPP equation, we apply Taylor's series on each term of the discritized scheme. Let us consider the following,

$$
\begin{aligned}
& u_{t}=u_{i}^{n+1}-u_{i}^{n} \\
& u_{t}=u(x, t+k)-u(x, t) \\
& \text { Apply Taylor's series } \\
& u_{t}=k u_{t}+\frac{k^{2}}{2} u_{t t}+\frac{k^{3}}{6} u_{t t}+\cdots
\end{aligned}
$$

above mentioned scheme with Taylor's series, we have

$$
E q .=\left(0.5 u_{t}-0.5 u(1-u)-0.5 u_{x x}\right) k+\frac{1}{8} u_{t t} k^{2}-0.5 u_{x x} h^{2}+\cdots
$$

Now principle part of the truncation error along with Equation (1). So first part of the above equation goes to zero if we consider Equation (1).

$$
\text { PPTE of } u=\frac{1}{8} u_{t t} k^{2}-0.5 u_{x x} h^{2}+\cdots
$$

Which show that this scheme is second order accurate in time and space, such as $O\left(k^{2}, h^{2}\right)$.

Stability:

We consider Von-Neumann stability analysis to explain on Lax-Wendroff scheme. Consider the scheme in the following way,

$$
u_{m}^{\star}=0.5\left(u_{i+1}^{n}+u_{i-1}^{n}\right)+0.5 \boldsymbol{R}_{1} \delta_{x}^{2} u_{m}^{n}+0.5 \alpha k u_{m}^{n}\left(1-u_{m}^{n}\right)
$$

Linear form of the above equation is,

$$
\begin{aligned}
& u_{m}^{\star}=0.5\left(u_{i+1}^{n}+u_{i-1}^{n}\right)+0.5 \boldsymbol{R}_{1} \delta_{x}^{2} u_{m}^{n}+0.5 \alpha k u_{m}^{n} \\
& (1-\text { Constant })
\end{aligned}
$$

According to Von-Neumann stability analysis as (5), (6) Note that,

$$
\begin{aligned}
U_{m}^{n+1} & =e^{\alpha(n+1) k} e^{i \beta m h} \\
U_{m}^{n-1} & =e^{\alpha(n-1) k} e^{i \beta m h} \\
U_{m+1}^{n} & =e^{\alpha(n) k} e^{i \beta(m+1) h} \\
U_{m-1}^{n} & =e^{\alpha(n) k} e^{i \beta(m-1) h}
\end{aligned}
$$

Also

$$
\begin{aligned}
& \delta_{x}^{2} U_{m}^{n}=-4 \sin ^{2}\left(\frac{\beta h}{2}\right)\left[e^{\alpha n k} e^{i \beta m h}\right] \\
& \delta_{x}^{2} U_{m}^{n+1}=-4 \sin ^{2}\left(\frac{\beta h}{2}\right)\left[e^{\alpha(n+1) k} e^{i \beta m h}\right]
\end{aligned}
$$


Apply above terms to Equation (1), we get the following

$$
\begin{aligned}
e^{\alpha k} & =1-4 \boldsymbol{R}_{1} \sin ^{2}\left(\frac{\beta h}{2}\right) e^{\alpha k / 2}+\alpha k(1-\text { Constant }) e^{\alpha k / 2} \\
& \Rightarrow\left|e^{\alpha k}\right|^{2}=1-\alpha^{2}\left(1-\alpha^{2}\right)\left(1-\sin ^{2}(\beta h / 2)\right)
\end{aligned}
$$

According to above result, the Von-Neumann stability criterion $\left|e^{\alpha k}\right| \leq 1$ is satisfied as long as $\alpha \leq 1$, or equivalently, as long as the CFL condition is satisfied. In this respect, the dissipative properties of the Lax-Friedrichs scheme are not completely lost in the Lax-Wendroff scheme but are much less severe [15]. The Lax-Wendroff scheme is a two-level scheme, but can be recast in a one-level form by means of algebraic manipulations [15]. This is clear from expressions where quantities at time-levels $n$ and $n+1$ only appear [15].

\subsection{Crank Nicolson Scheme}

Let us apply an implicit method to improve the accuracy. The implicit method we consider, is Crank Nicolson. By substituting the value of $u_{m}^{n}$ by average value of mesh points, the Crank Nicolson method is a finite difference method used for numerically solving linear and nonlinear partial differential equations [16] [17] [18]. It is implicit in time and can be written in form of an implicit Runge Kutta method, and it is numerically stable [16] [17] [18]. The method was developed by John Crank and Phyllis Nicolson in the mid 20th century [18]. For diffusion equations (and many other equations), it can be shown that, the Crank Nicolson method is unconditionally stable [19]. However, the approximate solutions can still contain (decaying) spurious oscillations if the ratio of time step $k$ times to the square of space step $h^{2}$, is large (typically larger than $1 / 2$ per Von-Neumann stability analysis). For this reason, whenever large time steps or high spatial resolution is necessary, the less accurate backward Euler method is often used, which is both stable and immune to oscillations [20]. In this method, consider the followings,

$$
\begin{aligned}
& u_{i}^{n}=\frac{u_{i}^{n+1}+u_{i}^{n}}{2} \\
& u_{t}=\frac{u_{i}^{n+1}-u_{i}^{n}}{k} \\
& u_{x x}=\frac{1}{h^{2}} \delta_{x}^{2}\left(\frac{u_{i}^{n+1}+u_{i}^{n}}{2}\right)
\end{aligned}
$$

discretization to one dimensional Fisher KPP equation,

$$
\begin{aligned}
u_{i}^{n+1}= & u_{i}^{n}+0.5 \boldsymbol{R}_{1}\left(u_{i+1}^{n+1}-2 u_{i}^{n+1}+u_{i-1}^{n+1}+u_{i+1}^{n}-2 u_{i}^{n}+u_{i-1}^{n}\right) \\
& +\frac{1}{4} \alpha k\left(u_{i}^{n+1}+u_{i}^{n}\right)\left(2-u_{i}^{n+1}-u_{i}^{n}\right) .
\end{aligned}
$$

\section{Accuracy to Crank Nicolson Scheme:}

To find accuracy of the CN scheme for Fisher-KPP equation,we apply Taylor's series on each term of the discritized scheme. Let us consider the following, 


$$
\begin{gathered}
u_{t}=u_{i}^{n+1}-u_{i}^{n} \\
u_{t}=u(x, t+k)-u(x, t) \\
\text { Apply Taylor's series } \\
u_{t}=k u_{t}+\frac{k^{2}}{2} u_{t t}+\frac{k^{3}}{6} u_{t t}+\cdots \\
u_{x x}=u_{i+1}^{n+1}-2 u_{i}^{n+1}+u_{i-1}^{n+1}+u_{i+1}^{n}-2 u_{i}^{n}+u_{i-1}^{n} \\
u_{x x}=u(x+h, t+k)-2 u(x, t+k)+u(x-h, t+k) \\
+u(x+h, t)-2 u(x, t)+u(x-h, t)
\end{gathered}
$$

Apply Taylor's series

$$
u_{x x}=2 h^{2} u_{x x}+k h^{2} u_{x x t}+\frac{h^{4}}{6} u_{x x x x}+\frac{h^{2} k^{2}}{2} u_{x x t t}+\frac{h^{4} k}{12} u_{x x x x t}+\cdots
$$

which implies

$$
\begin{aligned}
E q .= & \left(u_{t}-0.5 u \alpha(2-2 u)-u_{x x}\right) k+\left(0.5 u_{t t}-0.5 u_{x x t}+0.5 \alpha u u_{t}-0.25 \alpha u_{t}\right)(2-2 u) k^{2} \\
& -\frac{1}{12} k h^{2} u_{x x x x}+\left(\frac{1}{6} u_{t t t}+\cdots\right) k^{3}+\cdots
\end{aligned}
$$

Now principle part of the truncation error along with Equation (1). So first and second part of the above equation goes to zero if we consider Equation (1).

$$
\text { PPTE of } u=\left(\frac{1}{6} u_{t t t}+\cdots\right) k^{2}-\frac{1}{12} h^{2} u_{x x x x}+\cdots
$$

Which show that this scheme is second order accurate in time and space, such as $O\left(k^{2}, h^{2}\right)$.

Stability:

According to Von-Neumann stability analysis, we have linear form of the Equation (1) is,

$$
u_{m}^{n+1}-u_{m}^{n}=\frac{\boldsymbol{R}_{1}}{2}\left(\delta_{x}^{2} u_{m}^{n+1}+\delta_{x}^{2} u_{m}^{n}\right)+\frac{\alpha k}{2}\left(u_{m}^{n+1}+u_{m}^{n}\right)(1-\text { Constant })
$$

According to Von-Neumann stability analysis as (5), (6), after some simplifications, we reached at,

$$
\Rightarrow e^{\alpha k}=\frac{1-\sin ^{2}(\beta h / 2)}{1+2 \boldsymbol{R}_{1} \sin ^{2}(\beta h / 2)} .
$$

Hence the Von-Neumann stability condition is satisfied for all positive values of $\boldsymbol{R}_{1}$. This shows that $\mathrm{CN}$ scheme is unconditionally stable.

\subsection{Richardson Extrapolation Technique}

Techniques for obtaining more accurate solutions by using finite difference methods which have already been discussed, including reducing the truncation error by using central differences, using methods which minimize numerical damping and dispersion as well as using more accurate approximation to derivative boundary conditions [21] [22]. Other methods include,

1) reducing the size of grid spacing,

2) using higher order difference approximation, and 
3) reducing the discretisation error by extrapolation (Richardson).

Richardson extrapolation method, which was introduced by Richardson (1910) and later on called "deferred approach to the limit", may lead to considerable improvement of numerical results which solving the partial differential equation system by finite difference method. Applications of this method to equations solved on rectangular regions in space are described in Richardson and Gaunt (1927) and Salvadori (1951) [22]. The reduction of the error depends upon there being a reliable estimate of the discretisation error as a function of the grid spacing $h$. Let $\phi$ be the exact solution of the partial differential equation and $\phi^{1}, \phi^{2}$ the finite difference solutions at a particular point in the solution domain computed using grid spacing $h_{1}, h_{2}$ respectively [22] [23]. Richardsons extrapolation formula is as follows,

$$
N_{j}(h)=N_{(j-1)}\left(\frac{h}{2}\right)+\frac{N_{(j-1)}\left(\frac{h}{2}\right)-N_{(j-1)} h}{4^{(j-1)}-1}
$$

where $N_{j}(h)$ is the approximations which is even more accurate then the previous $N_{j-1}$ and $j=2,3, \cdots$. In our case we use $j=2$ to get the approximation formula $N_{2}(h)$ with truncation error $O\left(h^{4}\right)$. This gives more accurate and precise result which lead to convergence [23]. This method improvement the results upto fourth order accuracy in space. Keep in mind, accuracy in time still upto second order. Also this method hold the stable results. Clearly, this is a useful way of increasing the accuracy of the result at a given grid point without having to excessively increase the number of calculations required. This method may be of uncertain value near the boundaries which are not straight, such type of problems can be discussed with mixed boundaries with interpolation technique [23].

\section{Results}

Numerical computations have been performed by using the uniform grid [23]. For the test problem, the approximated and exact values of $u(x, t)$ and $U(x, t)$ have been given in Figure 1 with some fixed parameters such as $\alpha=1$, and $k=0.0001$ using FTCS. Figure 2 shows the results taken at different time to maintain stability. Figure 3 shows results by using Crank Nicolson scheme; comparison has been done with existence of exact solution. Figure 4 represents results from Richardson Extrapolation. These values has been taken at some typical grid point (TGP) as we presents in Table 1 and Table 2. Some critical values can be seen from the following data Table 1 and Table 2 at different location along $x$-axis. In Table 1 , we compare our results, calculated from Crank-Nicolson scheme with exact solution. Error can be seen from last column.

\section{Discussion}

In this paper, the solution of the Fishers equation is successfully approximated by a various numerical finite difference schemes. Two of them are explicit in nature such as FTCS and Lax-Wendroff. We have to pay attention to parameter $\boldsymbol{R}_{1}$, 


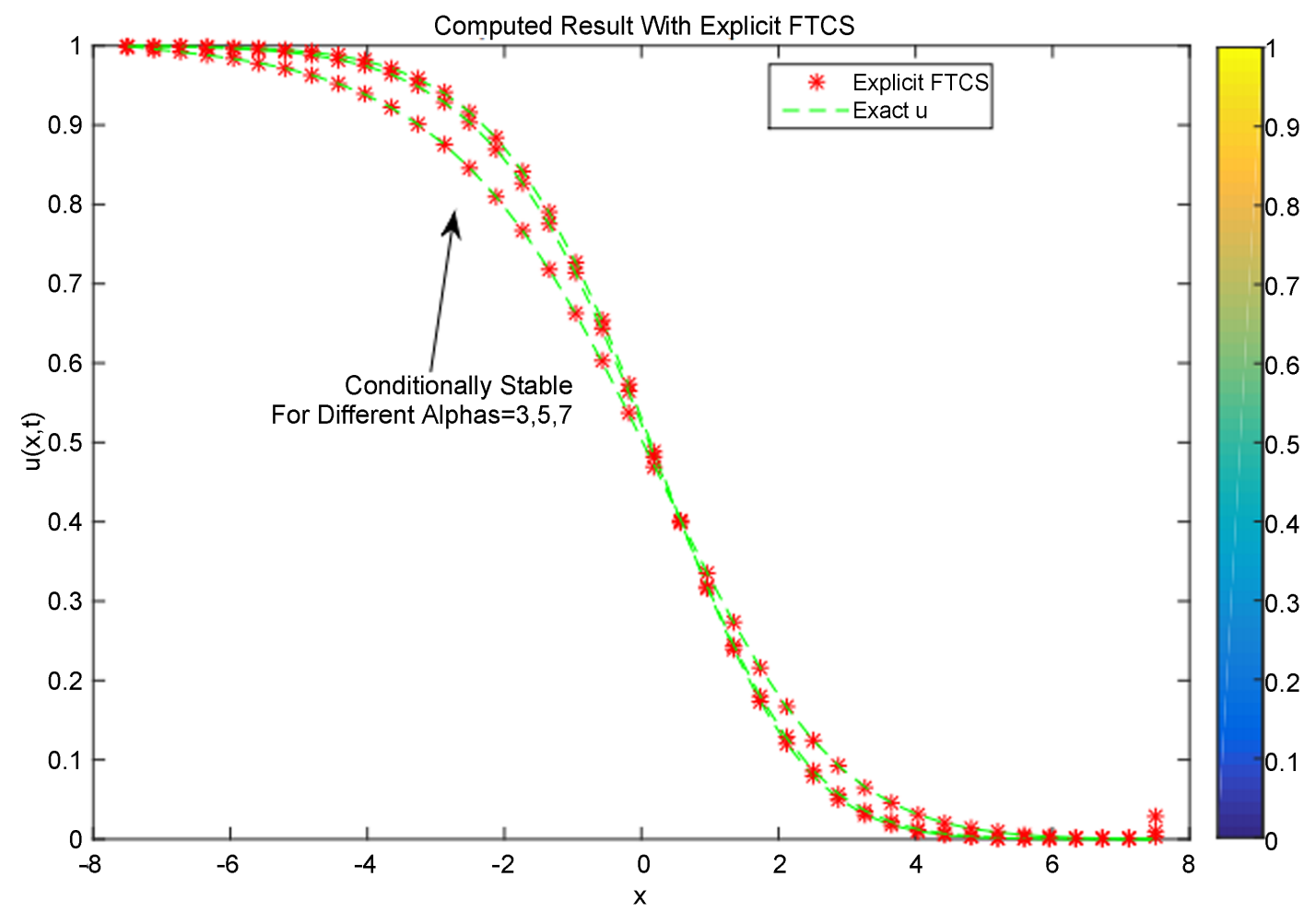

Figure 1. Figure shows results with FTCS, by fixing some parameters to be in range of stability. Due to explicit nature of the scheme, we choose $h$ and $k$ in such a way that $r \leq 1$.

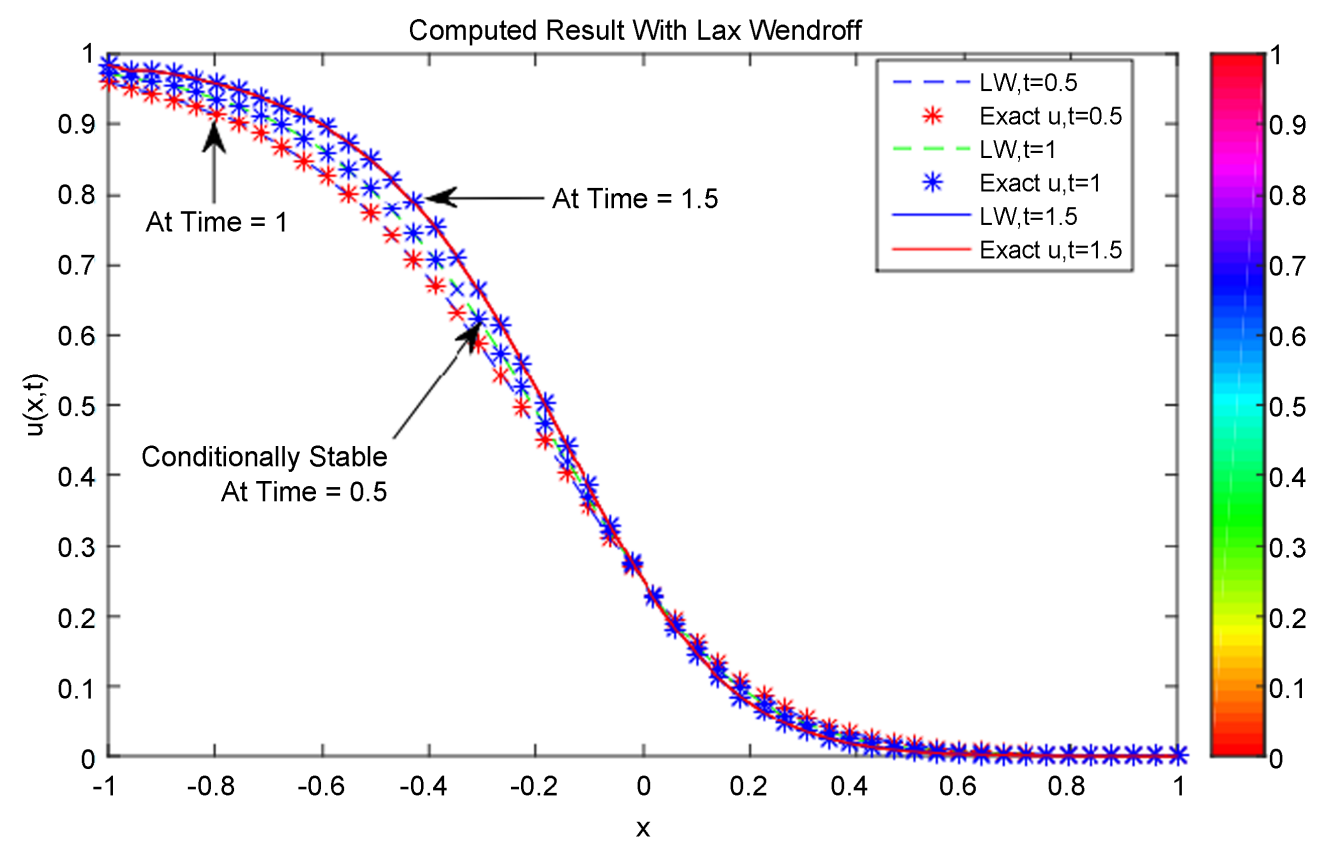

Figure 2. Figure shows results with Lax-Wendroff, by fixing some parameters to stabilize. Due to explicit nature of the scheme, we choose $h$ and $k$ in such a way that $r \leq 1$ with acceleration in computations. Results at different time levels, also compared with exact solution.

which can stabilize the results as we can see from Figure 1 and Figure 2. Such schemes on nonlinear one dimensional PDE, consistency, stability and convergence are more difficult to prove [24] [25] [26]. For instant, Von-Neumann's 


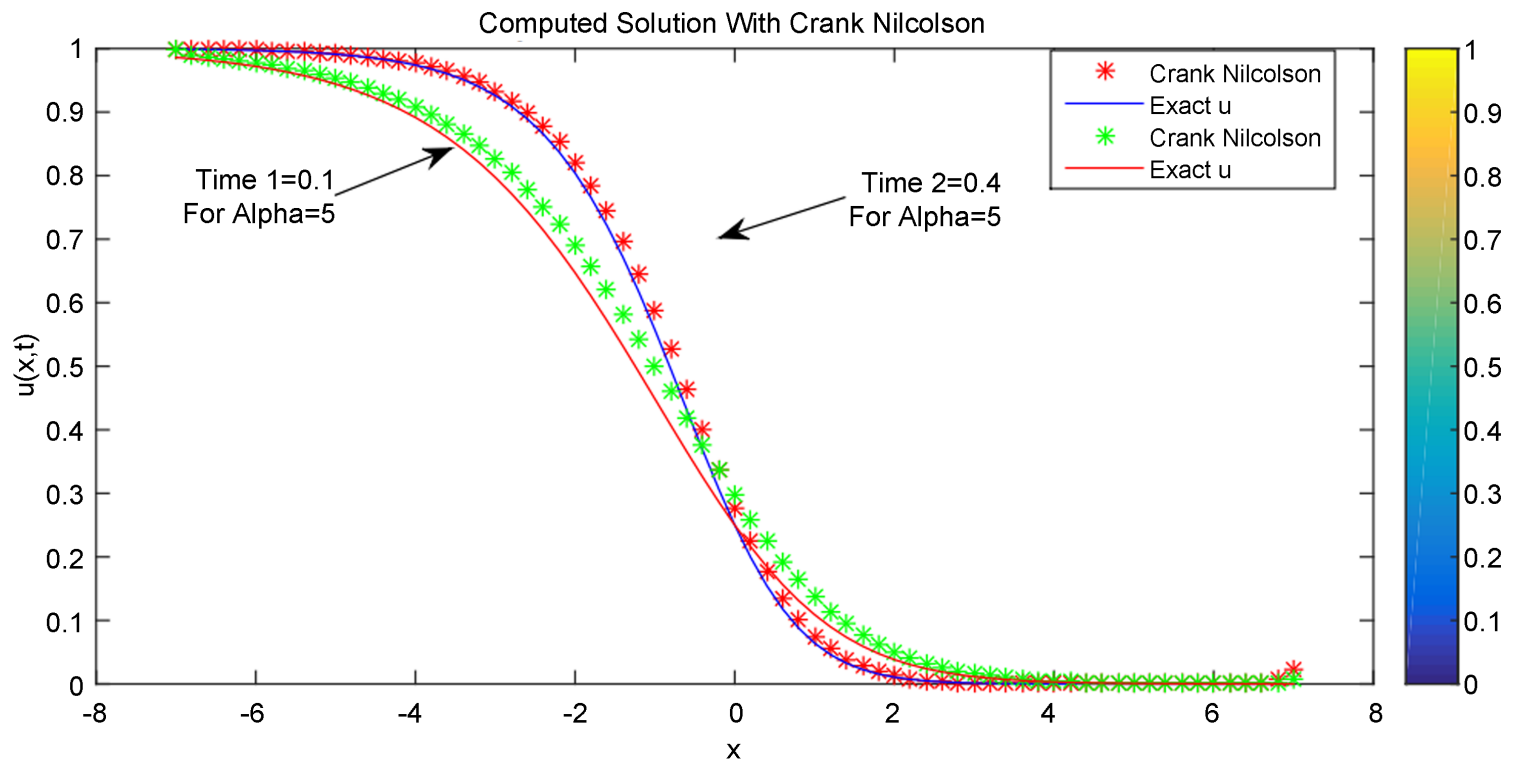

Figure 3. Shows results with implicit Crank Nicolson, different $\alpha$ and time level along comparison with exact solution.

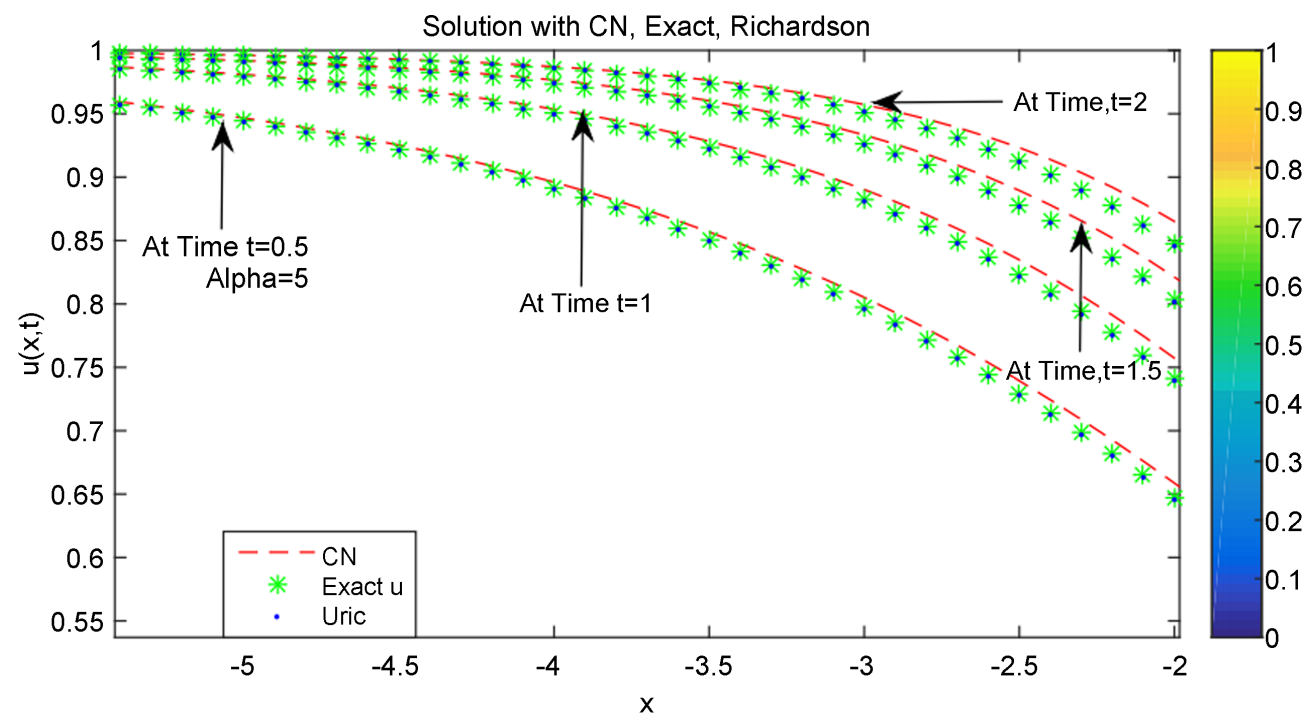

Figure 4. Shows results with Richardson Extrapolation, different time levels along comparison with exact solution.

Table 1. Estimates the results at different locations with Crank Nicolson scheme.

\begin{tabular}{ccccc}
\hline & & \multicolumn{3}{c}{$\Delta=0.0001$} \\
\hline$\alpha$ & Locations & $u_{\text {approximated }}$ & $U_{\text {Exact }}$ & Error $_{u-U}$ \\
\hline 1 & -4.6 & 0.987634663 & 0.98623750641 & 0.001397157 \\
& -3.9 & 0.973936808 & 0.97102606750 & 0.002910740499733 \\
& -2.2 & 0.852003299 & 0.83722482644 & 0.014778472550733 \\
& -1.3 & 0.671411264 & 0.64455730767 & 0.026853956325752 \\
& & & \\
& 0.027295815 & 0.02274615418 & 0.004549660819317 \\
& 0.9 & 0.007537831 & 0.00722501612 & $3.1281487779103 \mathrm{e}-04$ \\
\hline
\end{tabular}


Table 2. Estimates results from Richardson-Extrapolation with exact solution. Error can be seen from last column.

\begin{tabular}{ccccc}
\hline & & & \multicolumn{2}{c}{$\Delta=0.0001$} \\
\hline$\alpha$ & Locations & $u_{\text {approximated }}$ & $U_{\text {Exact }}$ & Error $_{u-U}$ \\
\hline 1.3 & -4.6 & 0.98623750641 & 0.986599027119808 & $3.6152070844 \mathrm{e}-04$ \\
& -3.9 & 0.97102606750 & 0.971912570950872 & $8.8650345060 \mathrm{e}-04$ \\
& -2.2 & 0.83722482644 & 0.8394970586880878 & 0.0022722322 \\
-1.3 & 0.64455730767 & 0.649302537205783 & 0.0047452295 \\
1.6 & 0.02274615418 & 0.022746146441480 & $7.7392030006 \mathrm{e}-09$ \\
6.9 & 0.00722501612 & 0.007225016102431 & $1.9777999550 \mathrm{e}-11$ \\
\hline
\end{tabular}

method of stability analysis can not be used other than locally, since it is only applied to linear finite difference schemes. In many cases, numerical experimentation, such as solving the finite difference schemes by using progressively smaller grid spacing and examining the behaviour of the sequence of the values of $u(x, t)$ obtained at given points, is the suitable method available with which to assess the numerical model [26]. The various methods of obtaining a finite difference numerical model corresponding to a particular mathematical model may result in either explicit or implicit finite difference schemes. Explicit schemes are conditionally stable and implicit schemes are unconditionally stable [26]. Two implicit schemes are also applied to improve accuracy, stability restrictions and consistency in solution. It can be observed that the computed results show excellent agreement with the exact solution. Our main purpose of this research to improve accuracy in result of Fisher KPP equation. At the end, we introduce Richardson extrapolation method; we observe improvement in accuracy and this method also stable and show excellent agreement with the exact solution. Accuracy in results are glanced from figures and tables.

\section{Acknowledgements}

The authors are gratefully acknowledge for support by Dr Muhammad Faheem Afzaal, Department of Chemical Engineering, Imperial College London and Vineet K. Srivastava, Scientist, ISTRAC/ISRO, Bangalore, India in understanding mathematical terms and algorithms.

\section{Conflict of Interest}

The authors do not have any conflict of interest in this research paper.

\section{References}

[1] Fisher, R.A. (1936) The Wave of Advance of Advantageous Genes. Annals of Eugenics, 7, 355-369. https://doi.org/10.1111/j.1469-1809.1937.tb02153.x

[2] Canosa, J.C. (1973) On a Nonlinear Diffusion Equation Describing Population Growth. IBM Journal of Research Development, 17, 307-313. https://doi.org/10.1147/rd.174.0307

[3] Arnold, R.A., Showalter, K. and Tyson, J.J. (1987) Propagation of Chemical Reac- 
tions in Space. Journal of Chemical Education, 64, 740-744. https://doi.org/10.1021/ed064p740

[4] Tuckwell, H.C. (1988) Introduction to Theoretical Neurobiology. Cambridge, UK: Cambridge University Press, Cambridge.

[5] Gazdag, J.G. and Canosa, J.C. (1974) Numerical Solutions of Fisher's Equation. Journal of Applied Probability, 11, 445-457. https://doi.org/10.1017/S0021900200096236

[6] Abdullaev, U.G. (1994) Stability of Symmetric Travelling Waves in the Cauchy Problem for the KPP Equation. Differential Equations, 30, 377-386.

[7] Logan, D.J. (1994) An Introduction to Nonlinear Partial Differential Equations. Wiley, New York.

[8] Evans, D.J. and Sahimi, M.S. (1989) The Alternating Group Explicit (AGE) Iterative Method to Solve Parabolic and Hyperbolic Partial Differential Equations. Annals of Numerical Fluid Mechanics and Heat Transfer, 2, 283-389.

https://doi.org/10.1615/AnnualRevHeatTransfer.v2.100

[9] Tang, S.T. and Weber, R.O. (1991) Numerical Study of Fisher's Equations by a Petrov-Galerkin Finite Element Method. Journal of the Australian Mathematical Society Series B, 33, 27-38. https://doi.org/10.1017/S0334270000008602

[10] Khaled, K.A. (2001) Numerical Study of Fisher's Diffusion Reaction Equation by the Sinc Collocation Method. Journal of Computational and Applied Mathematics, 137, 245-255.

[11] Ames, W.F. (1965) Nonlinear Partial Differential Equations in Engineering. Academic Press, New York.

[12] Ames, W.F. (1969) Finite Difference Methods for Partial Differential Equations. Academic Press, New York.

[13] Noye, J.N. (1981) Nonlinear Partial Differential Equations in Engineering. Conference in Queen's College, University of Melbourne, North-Holland Publishing Company, Australia.

[14] Mittal, R.C. and Kumar, S.K. (2009) Numerical Study of Fisher's Equation by Wavelet Galerkin Method. International Journal of Computer Mathematics, 3, 287 298. https://doi.org/10.1080/00207160600717758

[15] Mehdi, M.B. and Khojasteh, D.S. (2013) A Highly Accurate Method to Solve Fisher's Equation. Pramana Indian Academy of Sciences Journal of physics, 78, 335-346.

[16] Ablowitz, M.J. and Zeppetella, A.Z. (1979) Explicit Solutions of Fisher's Equation for a Special Wave Speed. Bulletin of Mathematical Biology, 41, 835-840. https://doi.org/10.1007/BF02462380

[17] Wasow, W.W. (1955) Discrete Approximation to Elliptic Differential Equations. Eitschrift Angew, Mathematical physics, 6, 81-97. https://doi.org/10.1007/BF01607295

[18] Schiesser, W.E. and Griffiths, G.W. (2009) A Compendium of Partial Differential Equation Models. Cambridge University Press, Cambridge. https://doi.org/10.1017/CBO9780511576270

[19] Whitham, G.B. (1974) Linear and Nonlinear Waves. John Wiley \& Sons, Hoboken.

[20] Babuska, I.B. (1968) Numerical Stability in Mathematical Analysis. IFIP Consress, Amsterdam, 11-23.

[21] Deghan, M.D., Asgar, H.A. and Mohammad, S.M. (2007) The Solution of Coupled Burgers Equations Using Adomian-Pade Technique. Applied Mathematics Computation, 189, 1034-1048. 
[22] Lax, P.D. and Wendroff, B.W. (1960) Systems of Conservation Laws. Communications on Pure and Applied Mathematics, 13, 217-237. https://doi.org/10.1002/cpa.3160130205

[23] Kanti, P.K. and Lajja, V.L. (2011) A Note on Crank-Nicolson Scheme for Burgers Equation. Applied Mathematics, 2, 888-899.

[24] Roache, P.J. (1972) Computational Fluid Dynamics. Hermosa, Albuquerque, 51179.

[25] Mazumder, S.M. (2015) Numerical Methods for Partial Differential Equations: Finite Difference and Finite Volume Methods. Academic Press, New York.

[26] Srivastava, V.K. and Tamsir, M.T. (2012) Crank Nicolson Semi Implicit Approach for Numerical Solution of Two Dimensional Coupled Nonlinear Burgers Equations. Journal of Applied Mechanics and Engineering, 17, 571-581.

Submit or recommend next manuscript to SCIRP and we will provide best service for you:

Accepting pre-submission inquiries through Email, Facebook, LinkedIn, Twitter, etc. A wide selection of journals (inclusive of 9 subjects, more than 200 journals)

Providing 24-hour high-quality service

User-friendly online submission system

Fair and swift peer-review system

Efficient typesetting and proofreading procedure

Display of the result of downloads and visits, as well as the number of cited articles

Maximum dissemination of your research work

Submit your manuscript at: http://papersubmission.scirp.org/

Or contactajcm@scirp.org 